\title{
Los otros hijos de la Malinche: historia y literatura heterodoxas sobra la migración mexicana
}

RODRIGO PARDO FERNÁNDEZ

UNIVERSIDAD MICHOACANA DE SAN NICOLÁS DE HIDALGO (MORELIA, MÉXICO)

ABSTRACT: The migratory flow of the Mexican population to the United States has created its own culture with a network of complex relations: economic, political, social, identity, that need to be studied from an interdisciplinary point of view. Thus, it is possible to understand the way in which literature and those who conceibe it as a cultural practice within the United States have created a space where the debate concerning identity, language and the experience of minorities holds a prominent place.

Keywords: migration, identity, Chicano literature, language.

RESUMEN: El flujo migratorio de la población mexicana a Estados Unidos se ha constituido como una cultura propia, con una red de relaciones complejas: económicas, políticas, sociales, identitarias, que necesita ser estudiada desde un punto de vista interdisciplinario y abierto. De este modo, es posible entender el modo en el que la literatura, y quienes la realizan como una práctica cultural específica en el seno de los Estados Unidos, se ha mostrado como un espacio donde la discusión en torno a la identidad, el lenguaje y la experiencia de las minorías se encuentra vigente de manera explícita.

Palabras clave: migración, identidad, literatura chicana, lenguaje.

\section{Introducción}

La literatura y la historia se conforman como dos discursos sobre el mundo que posibilitan su comprensión a partir de la escritura. Como otros discursos, son construidos a partir de una serie de convenciones sociohistóricas, lingüísti- 
cas y estéticas, pero de muchos modos hacen posible la digresión de la manera en que el statu quo pretende validarse. Este trabajo pretende realizar una lectura crítica e interdiscursiva de los textos que refieren las relaciones de los últimos dos siglos entre México y Estados Unidos de Norteamérica, a fin de mostrar las contradicciones de este fenómeno teniendo en cuenta su trasfondo político y económico, pero poniendo especial atención en los distintos seres humanos que representan ciertas prácticas identitarias que los definen como parte de las culturas de la región fronteriza.

\section{Historia de una frontera}

La migración mexicana a Estados Unidos es un fenómeno que tiene su origen en el Tratado de Guadalupe de 1848. Dicho acuerdo binacional significó no solo la escisión de un territorio, sino también el hecho documentado de que cien mil ciudadanos mexicanos (Cué Canovas, 1970: 47) permanecieron en los estados de Texas, Nuevo México y Alta California, en convivencia con cuarenta mil estadounidenses de origen diverso y cien mil indios, cuya presencia en esa geografía había sido alentada por diversas políticas estadounidenses, conducentes a promover las agresiones armadas de los pueblos nómadas contra poblaciones mexicanas de la zona fronteriza. Por parte del gobierno mexicano, el acuerdo de paz que culminó con el tratado se vio justificado, en los siguientes términos, por el político que sustituyó, en ausencia, a Antonio López de Santa Anna en la presidencia, y ejerció el cargo del 16 de septiembre al 13 de noviembre de 1847:

[Informe presidencial del 2 de febrero de 1848] Debido a grandes dificultades en que se hallaría el país, de sostener, con buen éxito, la guerra contra Estados Unidos, se accedió a ir a las propuestas de los comisionados de paz de esa nación. En consecuencia, se firmó, el 2 de febrero, el Tratado de Guadalupe Hidalgo, mediante el cual México cede a Estados Unidos, Texas, Alta California y Nuevo México. Los territorios cedidos no se pierden por la suma de 15000 000, sino por recobrar los puertos y ciudades invadidas; por la cesación definitiva de todos los males, de todo género de horrores [...] (De la Peña y Peña, 1997: 49)

Quienes permanecieron en los territorios ahora adscritos a otra nación no eran, sin embargo, un grupo homogéneo, sino una mezcla de indios, mestizos, negros, mulatos y criollos. De algún modo, «La cultura española, asimilada por una población que le diera expresión propia, dio características perdurables a esas regiones» (García Cantú, 1991: 104); pero también la presencia indígena contribuyó a esa conformación identitaria: por un lado, la mayoritaria presencia indígena, que en gran parte estaba conformada por indígenas del centro del país: tlaxcaltecas, nahuas, entre otros; y en menor medida por indios de Aridoamérica: hopis, pimas, yaquis, utes, apaches, comanches, navajos, pápagos, entre otros. 
Esta incidencia de indígenas provenientes del desarticulado imperio mexicano fue una de las razones que incidió en la aculturación de la zona en términos de la cosmovisión del pueblo proveniente de Aztlán, factor que a partir de los años 70 cobró gran relevancia para el Movimiento ${ }^{1}$ en razón de su necesidad de construir la identidad de los chicanos frente a la de otras minorías y frente a la postura de la hegemonía de raza blanca en el poder en los Estados Unidos.

En otro término, ha sido documentada la llamada hispanización de muchos mestizos y la asunción, sostenida con frecuencia, de una pretendida pureza de la población y de la cultura de origen hispánico en los territorios que en su momento pertenecieron a la Nueva España, tal y como lo señala Paredes (1993: 3-5). A este respecto, es posible hablar de dos formas de entender la cultura de origen español en el sur de Estados Unidos: la ya mencionada, considerada como la pervivencia de una cultura hispanoamericana aislada y por tanto sin contaminar, relacionada de manera estrecha con la tradición europea, tan favorable a la necesidad de establecer la identidad norteamericana con base en referentes identificables en el viejo continente; y en segundo lugar, como la manifestación de una cultura regional, a ambos lados del río Bravo/Grande, con rasgos compartidos y tradiciones comunes que es posible delimitar y poner en relación.

Estas lecturas diversas tienen un origen común, la escisión geográfica y las decisiones políticas que la siguieron: a mediados del siglo XIX el incumplimiento histórico de los artículos VIII, IX y X del Tratado de Guadalupe se concreta en una política oficial estadounidense basada en la intolerancia frente a las poblaciones mexicanas.

El abierto hostigamiento que sufrieron los mexicanos, las más de las veces violento, y el rechazo cultural, racial, religioso, en muchos sentidos, no hicieron otra cosa que coincidir con una postura ideológica promovida desde el ámbito del poder estatal, pero interiorizada también por una sociedad norteamericana pluricultural (si hablamos de un origen europeo común).

En muchos de los periódicos de la región se hacía propaganda contra México, sobre todo aludiendo a los territorios texanos y más al oeste de los ríos Sabinas y Arkansas.

Las incursiones repetidas -cada vez más violentas, más coordinadas- en territorio mexicano acabarían concretándose en la segunda intervención norteamericana, que se llevó a cabo entre 1846 y 1848.

1. Nombre bajo el cual se identificaron y agruparon movimientos sociales y contraculturales de distinta índole en Estados Unidos, que comprendían desde las protestas del ámbito rural y el Teatro Campesino de Luis Valdez, conformado por estudiantes universitarios, hasta las organizaciones de trabajadores y miembros de la comunidad académica. 
Un discurso como el de la Doctrina Monroe y «América para los americanos» (1823), ${ }^{2}$ además de las repetidas iniciativas legislativas estadounidenses llegaron a proponer la anexión del territorio del sur en su totalidad, y no solo cerca de 110.000 kilómetros cuadrados.

The senator Calhoun [...] has submitted many sound observations respecting the diversity of character, of races and of institutions, which exist between us and Mexico. (Sunseri, 1979: 94)

\section{Cultura hegemónica vs cultura del desarraigo}

A la fecha, el discurso políticamente correcto elude cualquier posible discriminación de la multitud de grupos étnicos comprendidos en el territorio estadounidense, pero siempre bajo la premisa de que, como señala Esperanza García y García,

[...] el punto de vista tradicional y prevaleciente en los Estados Unidos sigue siendo el de la asimilación [...] la sociedad tiene enclaves temporales de pluralismo cultural, asumiendo que este último es más notable en aquellas regiones geográficas en las que históricamente han existido patrones de segregación más importantes. (García y García, 2007: 235)

El pluralismo cultural de la sociedad norteamericana, construida a partir de migrantes, conlleva la construcción de guetos; construcción no solo material sino ideológica, discursiva, en términos de los rasgos identitarios y la necesidad de conformar un coto de poder en el que la comunidad específica, minoritaria, pueda sentir que es capaz de tener el control. En el seno de una sociedad hegemónica, dicho control es solo ilusorio o superficial, pero es el que ha hecho posible la idea de barrio, entre otras:

The Barrio represents a figurative borderland between the past and the future, a transitional space of internal transformations. [...] The barrio is a literary space many of the characters want to escape from and at the same time return to; but for most of them, the only way out left is to keep moving. The barrio is definitely a socially constructed public space. [...] The Latino barrio is usually the first space

2. Hay referencias específicas a que el discurso implicito era «El indio bueno es el indio muerto»; en palabras que se atribuyen a James M. Cavanaugh en la sesión de representantes de mayo de 1869: «I have never seen in my life a good Indian... except when I have seen a dead Indian» (Mieder, 1997: 138-139). 
recent immigrants inhabit precisely because of this need to be close to those akin. (Domínguez Miguela, 2005: 59-60)

Un problema añadido lo ha aportado el fenómeno migratorio, en tanto se mueve entre varios factores contradictorios, pero en los cuales subyace la intolerancia frente a los migrantes de origen mexicano o la negación de los otros, sea cual sea su origen, solo por su procedencia, etnia, cultura o lengua distinta. Entre otras, las variables que participan de la paradoja son la necesidad del mercado estadounidense de mano de obra barata; la miseria y los círculos económicos viciosos en los que se mueve una parte significativa de la población mexicana; la necesidad del gobierno de la república de mantener la migración, dado que los ingresos que percibe el país por parte de las remesas internacionales son uno de los principales aportes al Producto Interno Bruto. Intentando precisar con cifras el modo en que se ha visto incrementada la migración desde México hacia Estados Unidos, se hablaba a principios del siglo XX de un total que fluctuaba entre los cinco y los diez mil migrantes mexicanos; en contraparte, la OCDE (2010) calcula que cada año hay alrededor de trescientos mil mexicanos más, en su mayoría indocumentados, viviendo en Estados Unidos.

Estos datos se enlazan de manera significativa con la referencia a la diversidad cultural de los primeros chicanos, quienes permanecieron en territorio hostil desde 1848. Aún hoy la diversidad es la regla y no la excepción cuando se hace el intento de entender a los migrantes mexicanos como parte de un todo. El origen cultural y étnico de quienes se desplazan a la frontera en primer término y a Estados Unidos en un segundo paso -poniendo en riesgo su vida, pagando sumas exorbitantes a traficantes de personas- resulta de lo más variado y conlleva complejidades tan específicas como el hecho de que un hablante de lengua indígena se encuentre viviendo, como indocumentado, en un contexto hegemónico de lengua inglesa sin hablar siquiera español. La migración indígena se ha convertido en un fenómeno que sobrepasa los límites regionales e incide en el modo en el que las comunidades se ven a sí mismas y se relacionan con otras. La siguiente nota da cifras específicas:

La gente perteneciente a [los mixtecos] este pueblo tradicional mexicano que vive y trabaja en Estados Unidos equivale prácticamente a 80 por ciento de la población que aún radica en los nueve distritos mixtecos de Oaxaca, que asciende a 556 mil 250 personas. (La Jornada, 22 de abril, 2007)

De esta manera, a pesar de los esfuerzos por establecer una identidad clara que podría llamarse mexicanidad, si se tiene en cuenta el más de medio centenar de lenguas indígenas que aún perviven y la extensión de la República Mexicana, se podrá comprender que la migración hacia el vecino del norte conlleva espe- 
cificidades que no es fácil ubicar como un todo homogéneo. Lo mexicano, por otra parte, se entiende grosso modo de manera diversa a un lado o a otro de la frontera: perteneciente a México, el país, con un sentido racial, heredado del más desfasado positivismo decimonónico y ladino, que reniega del indígena; o bien como herencia del mestizaje, lo que conlleva reconocer a La Malinche como la madre de La Raza.

Para intentar comprender esta construcción de una cosmovisión contemporánea, no podemos restringir la cuestión únicamente a los autores chicanos, entre otras razones, por las recientes aproximaciones teóricas desde el ámbito editorial y la academia -políticamente correctas, acordes con una visión estatal y del aparato productivo que aparenta ser incluyente- que hablan de literatura hispana en los Estados Unidos (Stavans, 2011), relacionando a los escritores de origen dominicano, puertorriqueño, cubano, mexicano, centro o sudamericanos, migrantes o descendientes de ellos.

Hay otro factor que sustenta la anterior postura, relacionado con la coincidencia en perspectivas, en modos de reflexionar críticamente sobre sí mismos y sobre los otros en un país de diferencias socioculturales, de contrastes y claroscuros sociales, étnicos.

Teniendo en cuenta esta situación, es posible entender el modo en el que la literatura, y quienes la llevan a cabo como una práctica cultural específica en el seno de los Estados Unidos, se ha mostrado como un espacio donde la discusión en torno a la identidad, el lenguaje, la experiencia de las minorías, entre otros conceptos, se encuentra vigente de manera explícita.

\section{Narrativa de la migración}

El flujo migratorio de la población mexicana a Estados Unidos de Norteamérica se ha constituido como una cultura propia -dada su continuidad en el tiempo, su consolidación-, con una red de relaciones complejas: económicas, políticas, sociales, identitarias, que necesita ser estudiada desde un punto de vista interdisciplinario y abierto. Desde mediados del siglo XIX se ha desarrollado, como parte de esta cultura específica, binacional y bilingüe, con base en la confrontación y el sincretismo, una literatura propia de la migración: la frontera, el paso, la vida como indocumentado. Debe reconocerse a los textos narrativos de la frontera no solo como resultado de una tradición literaria concreta (mexicana o estadounidense, novelística o de otra índole), sino también proponer vías de comprensión de la literatura como una práctica cultural más compleja, relacionada con otros discursos y prácticas culturales, no necesariamente en la misma lengua o geografía. 
Teniendo en cuenta su experiencia como transgresores del statu quo, los migrantes trastocan un orden pretendidamente estable, pero que se basa en su esencia en la contradicción. Como ha señalado Rubén Martínez (2009: 127):

Es 1994. Ya no los llamamos refugiados, o comunistas. Ahora son ilegales, invasores, criminales, y siguen viniendo. América, tierra de inmigrantes, niega al inmigrante. En una temporada de furia política, la clase media contradice la señal inmigrante, e intenta borrar la conexión entre Los Ángeles y San Salvador, Los Ángeles y Guadalajara, que por supuesto es la conexión con Los Ángeles mismo. En verdad, el inmigrante nos contradecía a nosotros. El inmigrante era un recuerdo constante de la clase en la sociedad «sin clases», de la raza en la nación «sin raza». El inmigrante nos decía que no había frontera entre Estados Unidos y el resto del mundo, que no había excepción a la historia; nos mostró cuánto de nuestra cultura y nuestra política es moldeado por los viajes de migrantes, de profetas.

Más allá de los intentos de la literatura migratoria (mexicana o chicana; en español, inglés o pachuco) por conformar o encontrar una identidad propia, hay nuevos intentos de hablar del fenómeno como un problema compartido y no como una situación de excepción, ni mucho menos restringida a un grupo social en particular.

El fenómeno migratorio, y los textos de carácter literario construidos en torno, son más complejos que el mero relato de las peripecias del paso del río Bravo o la violencia asociada al tráfico de personas, armas y drogas; es un proceso cultural que se extiende en el tiempo y se filtra en todos los procesos sociales, políticos, económicos, colectivos e individuales, íntimos, de la población que se marcha al otro lado, pero también de la que se queda y de aquella que ya habitaba los, digámoslo así, territorios ocupados.

\section{La escritura identitaria}

Desde los estudios literarios debe entenderse la aparición reiterada de los discursos de ficción sobre la migración (siempre, de algún modo, testimoniales), como un fenómeno mucho más complejo, relacionado de manera íntima con lo social, lo ideológico, con el poder y la miseria, la idealización del sueño de vida americano y la pérdida de las raíces.

Porque de lo que se trata, al fin, es de la raíz, el origen, el afán de constituir (descubrir, recuperar) la identidad. La novela de Karen Yamashita, Tropic of Orange (1997), como toda la narrativa sobre la migración, sobre la frontera como herida y sobre los me-chicanos como pueblo, se conforma a partir de la idea de la raíz al aire, sin sustento: el des-arraigo. La organización del espacio y la constitución de un lugar propio (individual y colectivo) suceden en lo concreto 
y en lo imaginario. La narradora no es de origen mexicano pero se aproxima, con detallados pormenores sobre el paso (a través de la frontera) y referencias culturales específicas, a una composición detallada del México contemporáneo -lo cual exige al neófito lector documentarse para entender los referentes-. En esta perspectiva en la que el ejercicio narrativo pretende, y consigue, ubicarse en el lugar del otro, lo chicano es una forma de establecerse, de reconocer la identidad y el desamparo, el origen común (¿mestizo, en Gonzalo Guerrero y ZazilHá?, en estricto sentido, los padres de una nación que se conforma en el sincretismo) pero al mismo tiempo el extrañamiento. El siguiente fragmento pretende aproximar nuestra lectura a este espacio de lo ajeno en términos de un diálogo basado, más que en el absurdo, en la libre interpretación de los hechos históricos:

South of his dreams, it had been a long journey. He could remember everything. Here was a mere moment of passage. As he approached, he could hear the chant of the border over and over again: Catch'em and throw'em back. Catch'em and throw'em back. Catch'em and throw'em back. It was the beginning of the North of his dream, but they questioned him anyway. They held the border to his throat like a great knife. «What is your name?»

«Cristóbal Colón.»

«How old are you?»

«Quinientos y algunos años.»

«When were you born?»

«Doce de octubre de mil quatrocientos noventa y dos.»

«Where were you born?»

«En el nuevo mundo.»

«That would make you-»

«Post-Columbian.»

«You don't look post-Columbian. What is your business here?»

«I suppose you would call me a messenger».

«And what is your message?»

«No news is good news?»

«Is that a question? Say, do you speak English?»

«Yes».

«Where did you learn to speak English?»

«At Harvard University».

«So you've studied in the Us? Where?»

"At Harvard, at the School of Business. I was there at the same time as Carlos Salinas de Gortari. Then at Stanford University, in Economics, with Henrique Cardoso. Also at Columbia University with Fidel Castro; I did my thesis in political theory there, you see. And finally at Annapolis; what I studied there is a secret». «Where is your visa? Your passport?»

"Were you not expecting me? You had better consult your State Department, not to mention the side agreements with labor and the environment. I am expected. Me 
están esperando». He moved forward, slipping across as if from one dimension to another.

And the words came immediately, «SPEAK ENGLISH NOw!».

(Yamashita, 1997: 198-9)

Este fragmento remite a distintas problemáticas comunes al discurso migratorio: el paso aduanal, el cuestionamiento de la identidad en relación al conocimiento o desconocimiento de la lengua hegemónica, el origen identitario de lo latinoamericano en la lengua española «que descendió de los barcos», parafraseando a Carlos Fuentes.

Si bien el origen de la relación binacional, tal y como se ha referido brevemente al inicio, estuvo determinada por la violencia, el desencuentro y, en gran medida, esos siguen siendo los mecanismos que rigen la construcción de la cultura fronteriza, la narrativa de Yamashita aporta una visión posmoderna que, a partir del libre arbitrio, posibilita relacionar los espacios del pasado y las complejas relaciones del presente a través de un ejercicio lúdico y crítico de los criterios que establecen el modo de relacionarse los habitantes del norte y del sur. La contradicción entre el migrante miserable y el tecnócrata mexicano formado en universidades privadas de alto nivel saca a relucir el modo en el que las diferencias sociales no solo determinan el modo de relacionarse al interior de un país, sino que trascienden el espacio local y se vuelven conformación específica del modus operandi de los sujetos, concretos o literarios, que realizan la historia.

\section{Conclusión}

La lectura de un texto literario, de acuerdo con esta aproximación, va más allá de su identificación específica con un grupo o una tendencia; refiere a la construcción de una forma específica de relacionarnos con el mundo y de participar de los discursos que subyacen en las relaciones de poder, en este caso referidas a los migrantes de origen mexicano y su literatura; esto es, la narrativa que se aproxima a su situación buscando comprender su complejidad, pero al tiempo reconociendo sus limitaciones, sus carencias, el riesgo permanente de solo repetir reivindicaciones en vez de realizar un ejercicio intelectual y crítico para aportar una perspectiva distinta de las cosas.

Poner en tela de juicio la historia y la literatura en tanto discursos, sin pretensiones reivindicativas fuera de contexto pero tampoco con falsos paternalismos, es solo el punto de partida de un ejercicio que debemos continuar realizando para comprender mejor este proceso sociohistórico y el modo en el que la ficción lo transforma en algo diferente. 
La complejidad de la población de origen migrante (cada vez más propicia a establecer relaciones de toda índole con otras comunidades minoritarias, pero al mismo tiempo reacia al cambio como amenaza de desaparecer) ha hecho posible, desde 1848 hasta los inicios del siglo XXI, que aún hoy estemos discutiendo qué cosa es eso de literatura chicana, latina o hispana. Nuestra reflexión debe partir de la claridad de la perspectiva histórica, pero también de una cierta flexibilidad de cara a la producción de textos que cuesta cada vez más sujetar a los límites de los parámetros identitarios de una comunidad específica. La complejidad y aparente contradicción de la realidad que se configura en la narrativa de la frontera debe posibilitar la perspectiva autocrítica y la proyección de reflexiones que cuestionen la problemática social de este territorio binacional y de los sujetos que lo habitan o lo cruzan en pos de una incertidumbre.

\section{Referencias bibliográficas}

Cué Canovas, A. (1970): Estados Unidos y el México olvidado, México, Secretaría de Educación Pública.

De la Peña y Peña, M. (1997): «Informe (1848)» en Ampudia, R. (ed.) (1997): Los Estados Unidos de América en los informes presidenciales de México, México, Fondo de Cultura Económica. 49-50.

Domínguez Miguela, A. (2005): «Views of the Barrio in Chicano and Puerto Rican Narrative», American@. New Frontiers in Us Latino/a Culture and Literature, 3 (1): 59-73.

García Cantú, G. (1991): Idea de México, México, Conaculta/Fondo de Cultura Económica.

GARCÍA Y GARCÍA, E. (2007): El movimiento chicano en el paradigma del multiculturalismo de los Estados Unidos: de pochos a chicanos, hacia la identidad, México, Universidad Iberoamericana/Centro de Investigaciones sobre América del Norte/Universidad Nacional Autónoma de México.

Martínez, R. (2009): «Bendecido por las contradicciones: reflexiones sobre escribir en medio», Luvina, 57: 118-128.

Mieder, W. (1997): The Politics of Proverbs: from Traditional Wisdom to Proverbial Stereotypes, Wisconsin, University of Wisconsin Press.

OECD (2010): International Migration Outlook 2010, $<$ http://www.oecd.org/document /42/0,3746,en_2649_37415_45591593_1_ 1_1_37415,00.html [20-9-2012]>

PARedes, A. (1993): Folklore and Culture on the Texas-Mexican Border, Austin, Center for Mexican American Studies/University of Texas at Austin.

Stavans, I. (2011): The Norton Anthology of Latino Literature, New York, W. W. Norton \& Company. 
SunSERI, A. R. (1979): Seeds of Discord: New Mexico in the Aftermath of the American Conquest, 1846-1861, Chicago, Nelson-Hall.

Yamashita, K. T. (1997): Tropic of Orange, Minneapolis, Coffee House Press. 